ranged from 30 to $55 \mathrm{~mm}$ with $57 \%$ of the population having values greater than the normal range. Proximal RVOT ranged from 26 to $49 \mathrm{~mm}$ with $40 \%$ of the population above the normal range. $28 \%$ of the population had RVOT values greater than the proposed "major criteria" for ARVC. RV length ranged from 70 to $110 \mathrm{~mm}$ and RVDarea from 13 to $38 \mathrm{~cm}^{2}$ with values falling above ASE cut-offs in $69 \%$ and $59 \%$ of the population, respectively. When indexed (ratio scaling) for BSA proximal RVOT ranged from 13 to $25 \mathrm{~mm} / \mathrm{m}^{2}$ with $6 \%$ of the population meeting the major criteria for ARVC. Peak RV $\varepsilon$ ranged from -18 to $-41 \%$ and peak RV SRS from -0.75 to $-2.65 \mathrm{l} / \mathrm{s}$, consistent with normal ranges proposed by the ASE. RV diastolic deformation indices displayed marked individual variability with a dominant $\mathrm{SRE}^{\prime}$ (mean $\pm \mathrm{SD}=2.0 \pm 0.61 \mathrm{l} / \mathrm{s}$ ) and smaller SRA $(1.25 \pm 0.56 \mathrm{l} / \mathrm{s})$.

Conclusion RV dimensions in endurance athletes are higher than those proposed as "normal" and likewise may be consistent with the criteria for ARVC. Despite this enlargement, RV function in endurance athletes is preserved and therefore the role of RV strain imaging may provide additional diagnostic value in differentiating physiological from pathological adaptation.
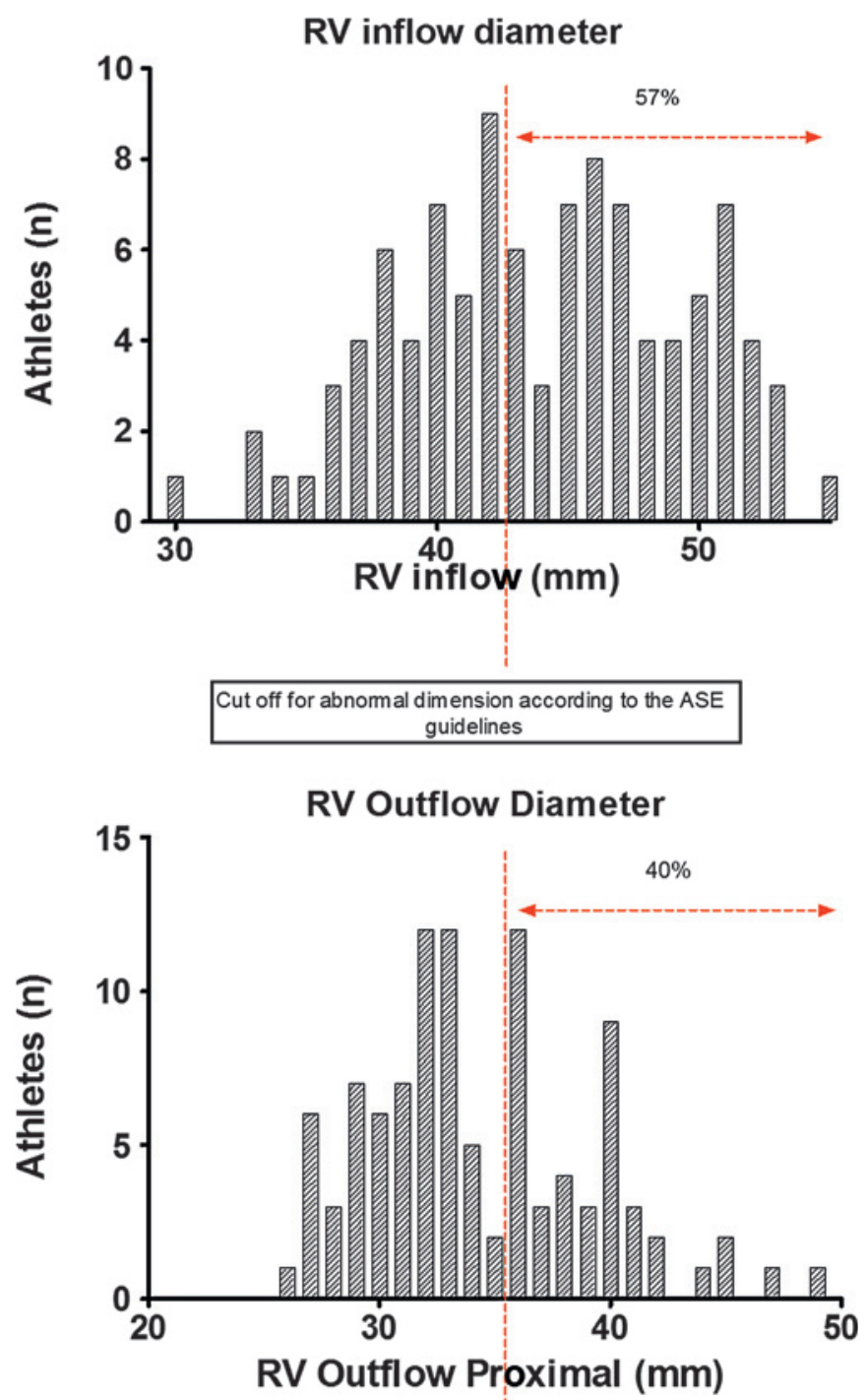

Cut off for abnomal dimension according to the ASE guidelines

Abstract 171 Figure 1
Abstract 171 Table 1

\begin{tabular}{llll}
\hline Parameter & Mean \pm SD (range) & $\begin{array}{l}\text { ASE Normal } \\
\text { Values }\end{array}$ & $\begin{array}{l}\text { Indexed (ratio } \\
\text { scaling) for BSA }\end{array}$ \\
\hline RVOT (mm) & $34 \pm 5(26$ to 49$)$ & $<35$ & $17 \pm 3(13$ to 25$) \mathrm{mm} / \mathrm{m}^{2}$ \\
RVDI (mm) & $44 \pm 5(30$ to 55$)$ & $<42$ & $22 \pm 3(15$ to 30$) \mathrm{mm} / \mathrm{m}^{2}$ \\
RV Length (mm) & $92 \pm 9(70$ to 110$)$ & $<86$ & $45 \pm 5(32$ to 61$) \mathrm{mm} / \mathrm{m}^{2}$ \\
RVDarea $\left(\mathrm{cm}^{2}\right)$ & $26 \pm 5(13$ to 38$)$ & $<25$ & $12.8 \pm 2(8.7$ to 17.6$)$ \\
RV $\varepsilon(\%)$ & $-27 \pm 6(-18$ to -41$)$ & -18 to -39 & $\mathrm{~N} / \mathrm{A}$ \\
RVSRS' (I/s) & $-1.53 \pm 0.43$ & 0.7 to 2.54 & $\mathrm{~N} / \mathrm{A}$ \\
& $(-0.75$ to -2.63$)$ & & \\
RVSRE' (I/s) & $2.0 \pm 0.61(0.87$ to 3.76$)$ & $\mathrm{N} / \mathrm{A}$ & $\mathrm{N} / \mathrm{A}$ \\
RVSRA' $^{\prime}(\mathrm{I} / \mathrm{s})$ & $1.25 \pm 0.56(0.28$ to 2.88$)$ & $\mathrm{N} / \mathrm{A}$ & $\mathrm{N} / \mathrm{A}$ \\
RVSR E' $/ \mathrm{A}^{\prime}$ & $1.89 \pm 1.03(0.49$ to 7.25$)$ & $\mathrm{N} / \mathrm{A}$ & $\mathrm{N} / \mathrm{A}$ \\
\hline
\end{tabular}

\section{INCREASING ANTI-OXIDANT CAPACITY REVERSES IRON OVERLOAD MEDIATED DYSFUNCTION IN CARDIOMYOCYTES}

doi:10.1136/heartjnl-2011-300198.172

${ }^{1} \mathrm{~F}$ R Millar, 'D T Baptista-Hon, ${ }^{2} \mathrm{~S}$ C O'Neill, ${ }^{1} \mathrm{M}$ E Díaz. ${ }^{1}$ University of Edinburgh, Edinburgh, UK; ${ }^{2}$ University of Manchester, Manchester, UK

Introduction Iron overload-cardiomyopathy (IOCM) is an increasing clinical problem worldwide. $70 \%$ of patients who receive compulsory blood transfusion die of IOCM, with increased susceptibility to arrhythmias and sudden death. We have previously found that iron exposure impairs cardiomyocyte $\mathrm{Ca}$ homeostasis. However, the cellular mechanisms responsible are unknown. Iron is known to participate in the Fenton reaction to produce reactive oxygen species (ROS), which mediate oxidative damage. We therefore tested the hypothesis that increasing the anti-oxidant capacity of cardiomyocytes, with the ROS scavenger Tempol, could be cardioprotective in the presence of iron.

Methods Single rat ventricular cardiomyocytes were loaded with fluo-3 to monitor intracellular Ca changes upon stimulation while bathed in control Tyrode solution and after adding ferrous iron (iron II). Sarcoplamic reticulum (SR) $\mathrm{Ca}$ load and sarcolemmal $\mathrm{Ca}$ extrusion rates were estimated during exposure to caffeine, which empties SR Ca stores. The ROS scavenger tempol was used to dissect ROS-mediated pathways from the direct effects of iron II on Ca handling. Data are provided as mean \pm SEM. Significance was tested using paired student $t$ test and defined as $p<0.05$.

Results Iron II exposure significantly increases systolic Ca transient amplitude (mean increase $82.8 \pm 21.8 \%, \mathrm{n}=9$ ) and causes spontaneous arrhythmogenic Ca release events (SACRE). These changes corresponded with increased SR $\mathrm{Ca}$ content (mean increase $21.0 \pm 5.7 \%, \mathrm{n}=8$ ), which is known to impact on systolic Ca release and spontaneous activity in cardiomyocytes. Sarcolemmal Ca extrusion rate was also significantly reduced upon iron II exposure (measured as the rate of fall of the caffeine response; mean decrease $48.7 \pm 5.5 \%, \mathrm{n}=8$ ), consistent with an overall gain of $\mathrm{Ca}$ by the cardiomyocyte. The onset of these Ca disruptions was significantly delayed in the presence of the ROS scavenger tempol $(p<0.001)$. Without tempol, SACRE onset occurred after $6.9 \pm 0.6 \mathrm{~min}(\mathrm{n}=22)$ following iron II exposure. The same manoeuvre in in tempol delayed the onset of SACRE to $17.8 \pm 1.8 \mathrm{~min}(\mathrm{n}=7)$. Furthermore, increasing ROS scavenging reversed the increase in systolic $\mathrm{Ca}$ transient amplitude, as well as SACRE upon washout of iron II. In contrast, in cardiomyocytes not exposed to tempol, the effects of iron II were irreversible.

Conclusions Our data show that iron II disrupts cardiomyocyte Ca handling. This is mediated via inhibition of sarcolemmal Ca extrusion, leading to SR Ca overload and SACRE. These are the initiators of most fatal non-reentrant arrhythmias and cardiac sudden death in 
experimental models. The observed effects are partly due to iron IImediated oxidative damage. This was confirmed by the presence of a ROS scavenger delaying the onset of the effects of iron II, and crucially rendering the effects reversible upon iron-washout. These effects of tempol suggest a novel therapeutic target for the treatment of IOCM patients.

\section{A GENERIC METHOD TO ASSESS THE ADEQUACY OF INDIVIDUAL MATERNAL CARDIAC RESERVE TO TOLERATE THE DEMANDS OF PREGNANCY AND LABOUR}

doi:10.1136/heartjnl-2011-300198.173

${ }^{1} \mathrm{D}$ Barker, ${ }^{2} \mathrm{~N}$ Lewis, ${ }^{2} \mathrm{G}$ Mason, ${ }^{2} \mathrm{~L}$ B Tan. ${ }^{1}$ Liverpool Heart and Chest Hospital, Liverpool, UK; ${ }^{2}$ Leeds General Infirmary, Leeds, UK

Introduction Clinicians often feel apprehensive when managing pregnant patients with heart disease. To complement current evaluation, we have developed a new method of directly assessing the individual patient's cardiac functional reserve through stress testing. Pregnant mothers with and without heart disease were studied to test the hypothesis that pregnant cardiac patients who possess cardiac reserve equivalent to that of controls can tolerate the usual demands of pregnancy, labour and puerperium.

Methods Fifty-one pregnant women with heart disease (mean age

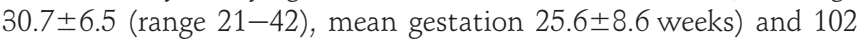
healthy pregnant women (mean age 31.4 \pm 5.0 , (range 19-41), mean gestation $25.1 \pm 9.2$ weeks) underwent maximal symptom-limited treadmill cardiopulmonary exercise testing. Fifty-nine non-pregnant women (mean age 32.7 \pm 5.1 (range 20-41) years) were similarly tested and used as a control group. Cardiac output (CO) was measured at peak exercise using the $\mathrm{CO}_{2}$ re-breathing method.
Cardiac power output (CPO) was calculated as the product of $\mathrm{CO}$ and mean arterial pressure. A composite endpoint including maternal death, fetal death, emergency caesarean section for maternal distress and significant morbidities was determined.

Results All tests were performed without significant complications. Employing data from a previous study of haemodynamics during labour in healthy women, the mean CPO required during peak labour is $2.6 \mathrm{~W}$. This value was adopted for investigation as the minimum required for an average woman to cope with the circulatory demands of normal labour. The healthy controls had a mean peak $\mathrm{CPO}(\mathrm{PkCPO})$ of $3.79 \pm 0.6 \mathrm{~W}$ and all non-pregnant women had $\mathrm{PkCPO}$ exceeding $2.6 \mathrm{~W}$. The majority of heart disease patients were able to achieve PkCPO values overlapping their healthy counterparts. Only a small proportion of the cardiac patients had $\mathrm{PkCPO}$ values lower than the $2.6 \mathrm{~W}$ cutoff. Women were significantly more likely to have uncomplicated pregnancy, labour and puerperium if able to achieve $\mathrm{PkCPO}>2.6 \mathrm{~W}$ (OR 8.1, 95\% CI 1.8 to 37.0, $\mathrm{p}=0.023$ ). Pregnant women in NYHA class I had PkCPO values indistinguishable from controls (mean $3.98 \pm 0.77 \mathrm{~W}, \mathrm{NS}$ ); whereas symptomatic pregnant women had significantly lower values (mean 3.15 $\pm 0.71 \mathrm{~W}, \mathrm{p}<0.005)$.

Conclusions Direct measurement of cardiac functional reserve capacity can be performed by maximal cardiopulmonary exercise testing with non-invasive assessment of $\mathrm{PkCPO}$, which can be safely undertaken during pregnancy. A cutoff value of PkCPO 2.6 W was identified as the lower limit for healthy women, corresponding to that required for normal labour. Most cardiac patients studied had PkCPO values comfortably above this cutoff, and all asymptomatic (NYHA I) and low risk cardiac patients had PkCPO values similar to controls. Measurement of PkCPO allows pregnant patients to be further classified into those with adequate vs limited cardiac reserve, supplementary to existing risk stratification methods. 